\title{
Antimicrobial activity of menthol modified nanodiamond particles
}

Volodymyr Turcheniuk, Viktoria Raks, Rahaf Issa, Ian R. Cooper, Peter J. Cragg, Roxana Jijie, Nicoleta Dumitrascu, Lyuba I. Mikhalovska, Alexandre Barras, Vladimir Zaitsev, Rabah Boukherroub, Sabine Szunerits

\begin{abstract}
Advances in nanotechnology have seen the development of several microbiocidal nanoparticles displaying activity against biofilms. These applications benefit from one or more combinations of the nanoparticle properties. Nanoparticles may indeed concentrate drugs on their surface resulting in polyvalent effects and improved efficacy to fight against bacteria. Nanodiamonds (NDs) are among the most promising new materials for biomedical applications. We elucidate in this paper the effect of menthol modified nanodiamond (ND-menthol) particles on bacterial viability against Grampositive (Staphylococcus aureus) and Gram-negative (Escherichia coli) bacteria. We show that while ND-menthol particles are non-toxic to both pathogens, they show significant antibiofilm activity. The presence of ND-menthol particles reduces biofilm formation more efficiently than free menthol, unmodified oxidized NDs and ampicillin, a commonly used antibiotic. Our findings might be thus a step forward towards the development of alternative non antibiotic based strategies targeting bacterial infections.
\end{abstract}

Keywords

Nanodiamond particles; Menthol; Functionalization; Antimicrobial activity; Biofilm inhibition

\section{Introduction}

Bacterial infections are one of the most serious risks to public health. Extensive efforts have been devoted to develop rapid and sensitive methods for the detection, identification of the pathogen strain at hand and for destruction or inhibition of pathogens and their actions [1], [2], [3] and [4]. Despite considerable recent progress in the understanding of the mechanisms underlying bacterial infections, and in the development of nanostructured materials displaying antibacterial properties and activity against biofilms [1], [5], [6], [7] and [8], the quest to design and fabricate new antibacterial nanostructures remains a high research priority. Nanoparticles have been considered as affective solution to fight against bacterial infections [4], [9], [10], [11], [12], [13], [14] and [15]. Such nanostructures allow the concentration of antibacterial agents and functions on their surface to deliver polyvalent effects. Some materials such as silver are themselves antibacterial and can result in enhanced efficacy [16], [17] and [18]. Although recent studies using silver nanoparticles appear promising [19], the antimicrobial activity is highly dependent upon their size and shape, with smaller particles demonstrating greater activity [20] and [21]. More recently concerns about the cytotoxic effects of silver nanoparticles against human cells have been voiced [22]. Moreover, 
practical applications of Ag NPs are often hampered by the aggregation and loss of antibacterial activity [23].

Among the potential alternatives to these compounds are nanodiamond particles (NDs). One of the advantages of NDs over other carbon-based materials such as fullerenes and carbon nanotubes is that they are completely inert, optically transparent, biocompatible and can be functionalized in many ways depending on their intended ultimate application [24], [25], [26], [27], [28], [29], [30] and [31]. Although their in vivo toxicity depends on their particular surface characteristics [32], ND particles do not induce significant cytotoxicity in a variety of cell types [32], [33], [34] and [35] and have been used in a variety of biomedical applications. More recently, the antibacterial activity of diamond particles [36], [37] and [38] and their potential to interfere with biofilm formation [1] have been highlighted. The underlying anti-adhesive strategy proposed was based on the interfering with type 1 fimbriae-mediated mannose recognition events [39]. Such biofilm disrupting activity had not been observed previously for other glyco-nanoparticles (glyco-NPs) such as glycofullerenes, goldbased glyco-NPs or for other multivalent mannose-derived molecules [2] and [40]. Wehling et al. have concluded that the bactericidal activity of diamond particles is directly linked to their surface chemistry, being thus the driving force of antibacterial effects [37].

In this paper, we investigate the antimicrobial properties of ND particles modified with menthol (Fig. $1 \mathrm{~A}$ ) and their potential to inhibit the growth of Gram-positive (Staphylococcus aureus) and Gramnegative (Escherichia coli) bacteria.

(A)

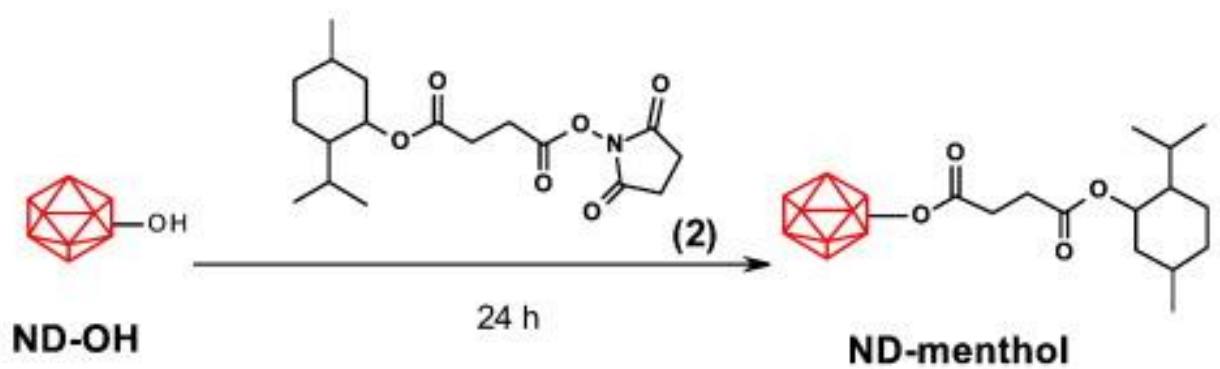

(B)

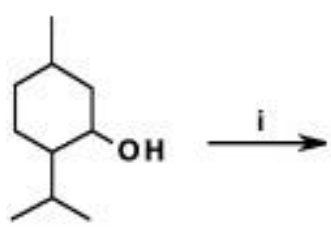<smiles>CC1CCC(C(C)C)C(OC(=O)CCC(=O)O)C1</smiles>

(1)<smiles>CC#CC1CCC(C)CC1OC(=O)CCC(=O)ON1C(=O)CCC1=O</smiles>

(2)

Fig. 1. (A) Schematic illustration of the stepwise chemical functionalization of nanodiamond particles (ND) to give the target ND-menthol cluster; (B) synthetic route to menthol derivative (2): (i) succinic anhydride, 4-DMAP, $\mathrm{CHCl} 3$, reflux, overnight, 84\%; (ii) NHS, DCC, $\mathrm{CH} 2 \mathrm{Cl} 2$, r.t, overnight, $95 \%$. 
Menthol, also known as mint camphor, is a naturally occurring cyclic terpene alcohol of plant origin, which has been used since antiquity for medicinal purposes [41], [42] and [43]. It is a major constituent in the essential oils of Mentha canadensis L. (cornmint) and peppermint possessing wellknown cooling characteristics and a residual minty smell of the oil remnants from which it was obtained. Several studies have additionally demonstrated that next to the effect of sensory parameters, menthol has antibacterial and antifungal activities [44] and [45]. However, the antimicrobial activity was reported to be lower compared to antibiotics such as penicillin [46]. More recently, Imbert and co-workers showed the in vitro activity of terpenes, including that of menthol, against Candida biofilms [47]. We show here that ND-menthol particles affect moderately S. aureus and $\mathrm{E}$. coli growth in a concentration dependent manner. In addition, the ND-menthol particles are found to inhibit $\mathrm{S}$. aureus and E. coli-driven biofilm growth significantly.

\section{Experimental}

\subsection{Materials}

Hydroxyl-terminated nanodiamond (ND-OH) particles were purchased from International Technology Center (Raleigh, NC, USA) and exhibit a primary average particle size of $4.0 \mathrm{~nm}$. (1R,2S,5R)-(-)-menthol, 4-dimethylaminopyridine (4-DMAP), succinic anhydride, hexane (Hex), dichloromethane $(\mathrm{CH} 2 \mathrm{Cl} 2)$, chloroform $(\mathrm{CHCl} 3)$, ethyl acetate (EtOAC), $\mathrm{N}, \mathrm{N}^{\prime}$-dicyclohexylcarbodiimide (DCC) and N-hydroxysuccinimide (NHS) were purchased from Sigma-Aldrich and used as received. All microbiological media, equipment and the ampicillin were purchased from Fisher Scientific (UK).

\subsection{Synthesis of menthol derivative (2)}

\subsubsection{Succinic acid mono-(2-isopropyl-4-methyl-cyclohexyl) ester (1)}

To the solution of menthol $(0.8 \mathrm{~g}, 5 \mathrm{mmol})$ in chloroform, 4-DMAP $(0.31 \mathrm{~g}, 2.5 \mathrm{mmol})$ and succinic anhydride $(1.02 \mathrm{~g}, 10 \mathrm{mmol})$ were added. This mixture was stirred overnight under reflux. After solvent evaporation, the crude product was purified using flash column chromatography with Hex:EtOAc as eluent to give white crystals $1.1 \mathrm{~g}$ (84\%). 1H NMR (300 MHz, CDCl3) $\delta 12.2-11.1$ (broad, 1H) 4.77-4.68 (m, 1H), 2.67-2.55 (m, 4H), 2.05-1.95 (m, 1H), 1.91-1.79 (m, 1H), 1.74-1.62 $(\mathrm{m}, 2 \mathrm{H}), 1.55-1.33(\mathrm{~m}, 2 \mathrm{H}), 1.1-0.8(\mathrm{~m}, 10 \mathrm{H}), 0.78-0.7(\mathrm{~d}, 3 \mathrm{H})$.

\subsubsection{Succinic acid 2,5-dioxo-pyrrolidin-1-yl ester 2-isopropyl-4-methyl-cyclohexyl ester (2)}

To the solution of acid (1) $(0.5 \mathrm{~g}, 1.9 \mathrm{mmol})$ in dichloromethane, $\mathrm{N}, \mathrm{N}^{\prime}$-dicyclohexylcarbodiimide $(0.4$ $\mathrm{g}, 1.9 \mathrm{mmol}$ ) and $\mathrm{N}$-hydroxysuccinimide $(0.22 \mathrm{~g}, 1.9 \mathrm{mmol})$ were added and the mixture was stirred overnight at room temperature. After filtration and solvent evaporation a white powder was obtained, yield: 95\%. $1 \mathrm{H} \mathrm{NMR} \mathrm{(300} \mathrm{MHz,} \mathrm{CDCl3)} \delta 4.77-4.68(\mathrm{~m}, 1 \mathrm{H}), 2.85-2.81(\mathrm{~s}, 4 \mathrm{H}), 2.54-2.46$ $(\mathrm{m}, 4 \mathrm{H}), 2.01-1.94(\mathrm{~m}, 1 \mathrm{H}), 1.88-1.76(\mathrm{~m}, 1 \mathrm{H}), 1.73-1.62(\mathrm{~m}, 2 \mathrm{H}), 1.55-1.33(\mathrm{~m}, 2 \mathrm{H}), 1.22-0.82(\mathrm{~m}$, $10 \mathrm{H}), 0.75-0.7(\mathrm{~d}, 3 \mathrm{H})$.

\subsection{Preparation of menthol-modified NDs (ND-menthol)}


Menthol derivative (2) (10 mM), was dissolved in $5 \mathrm{~mL}$ water and added to a suspension of ND-OH particles in water (10 mg in $5 \mathrm{~mL}$ ). The solution was stirred at room temperature for $24 \mathrm{~h}$ under nitrogen. The resulting ND-menthol particles were isolated by centrifugation at $10,000 \mathrm{rpm}$, purified through four consecutive wash/centrifugation cycles at 10,000 rpm with ethanol, and water and finally oven dried at $50^{\circ} \mathrm{C}$ for $24 \mathrm{~h}$.

\subsection{Characterization}

\subsubsection{FTIR spectroscopy}

Fourier transformed infrared (FTIR) spectra in transmission mode were recorded using a ThermoScientific FTIR instrument (Nicolet 8700 ) with a resolution of $4 \mathrm{~cm}-1$. Dried ND powder (1 $\mathrm{mg}$ ) was mixed with $\mathrm{KBr}$ powder $(100 \mathrm{mg})$ in an agate mortar. The mixture was pressed into a pellet under 10 ton load for 2-4 min, and the spectrum was recorded immediately. Sixteen accumulative scans were collected. The signal from a pure $\mathrm{KBr}$ pellet was subtracted as a background.

\subsubsection{Particle size measurements}

ND suspensions ( $20 \mu \mathrm{g} \mathrm{mL}-1)$ in water were sonicated. The particle size of the ND suspensions was measured at $25^{\circ} \mathrm{C}$ using a Zetasizer Nano ZS (Malvern Instruments S.A., Worcestershire, U.K.) in $173^{\circ}$ scattering geometry and the zeta potential was measured using the electrophoretic mode.

\subsubsection{Transmission electron microscope (TEM)}

TEM measurements were performed in a FEI Tecnai G2 20 equipped with EDS micro-analysis, Gatan energy filter (EELS), electron precision and tomography.

\subsubsection{Thermogravimetric analysis (TGA)}

Thermogravimetric analysis (TGA) measurements were made in Al2O3 crucibles in an atmosphere of nitrogen at a heating rate of $10^{\circ} \mathrm{C} \mathrm{min}-1$ using a TA Instruments $Q 50$ thermogravimetric analyzer.

\subsection{Antimicrobial assays}

\subsubsection{Bacterial biofilm formation}

Overnight cultures of E. coli (NCTC 8196) and S. aureus (NCTC 6571) were prepared by inoculating 10 $\mathrm{mL}$ of nutrient broth and incubating at $37^{\circ} \mathrm{C}$ for $18 \mathrm{~h}$ on a rotary shaker (Stuart Scientific) at 120 rpm. After incubation, the cultures were diluted to OD600 of 0.1 in nutrient broth at a final volume of $100 \mathrm{~mL}$, and a 96-well plate was set up as follows. Each assay sample required 6 wells, with another 6 wells for a negative control/blank, which consisted of $125 \mu \mathrm{L}$ of sterile nutrient broth. Next, $110 \mu \mathrm{L}$ of culture plus $15 \mu \mathrm{L}$ of PBS was pipetted into the next 6 wells to act as the positive growth control. In the remaining wells, the assay was set up as follows: $110 \mu \mathrm{L}$ of culture and $15 \mu \mathrm{L}$ of the appropriate NDs and ampicillin solutions. Sterile nutrient broth and NDs and sterile nutrient broth and ampicillin were also set up in sets of six wells each to act as controls. The 96-well plate was incubated at $37^{\circ} \mathrm{C}$ for $24 \mathrm{~h}$ with no agitation. After $24 \mathrm{~h}$, the plate was removed from the 
incubator, and the supernatant of each well was removed by pipetting. The plates were subsequently allowed to dry for 15-20 min in a Class Two Microbiological Hood. Next, $125 \mu \mathrm{L}$ of PBS was transferred into each well in order to remove any non-adhering cells. The PBS was then removed by pipetting, and the plates were again allowed to dry in the Class Two Microbiological Hood for $15 \mathrm{~min}$. All wells were stained by adding $150 \mu \mathrm{L}$ of $0.9 \%$ crystal violet in sterile reverse osmosis water, and the plates were incubated at room temperature for $15 \mathrm{~min}$. After this time, the stain was removed by pipetting, and excess dye was washed off by manually adding and then removing $165 \mu \mathrm{L}$ of PBS. To solubilize the crystal violet, $175 \mu \mathrm{L}$ of $95 \%$ ethanol was added to each well, and the plates were incubated at room temperature for $30 \mathrm{~min}$. The bacterial cell density was determined by the optical density of each well at $595 \mathrm{~nm}$, using the negative control wells as blanks.

\subsubsection{Viability of bacterial biofilms}

The LIVE/DEAD ${ }^{\circledR}$ BacLight $^{\mathrm{TM}}$ assay kit was used to determine the effect of ND-menthol $(100 \mu \mathrm{g} / \mathrm{mL})$ on bacterial cell viability. Overnight cultures of E. coli and S. aureus were prepared by inoculating 10 $\mathrm{mL}$ of nutrient broth and incubating at $37^{\circ} \mathrm{C}$ for $18 \mathrm{~h}$ on a rotary shaker (Stuart Scientific) at 120 $\mathrm{rpm}$, and subsequently diluted to OD600 of 0.01 in nutrient broth. ND-menthol $(100 \mu \mathrm{g} / \mathrm{mL})$ was suspended in sterile nutrient broth and added to respective cultures. The cultures were incubated in the presence of ND-methanol for $3 \mathrm{~h}$ (logarithmic growth phase) at $37^{\circ} \mathrm{C}$ at $120 \mathrm{rpm}$. After incubation, the cultures were centrifuged at $10,000 \mathrm{rpm}$ for $5 \mathrm{~min}$, and the supernatant solution was discarded. The resulting cell pellets were washed with $1.5 \mathrm{~mL}$ sterile PBS, and centrifuged at 10,000 rpm for $5 \mathrm{~min}$, three times to remove media constituents that may interfere with the stain. After each wash the supernatant was discarded and the pellet collected. After the final wash in PBS, the cells were re-suspended in $1 \mathrm{~mL}$ PBS. On ice, equal volumes of component $A$ and component $B$ of the LIVE/DEAD ${ }^{\circledR}$ BacLight $^{\text {TM }}$ stains were added to the cell suspensions and thoroughly mixed by vortexing. Next, $3 \mu \mathrm{L}$ of stain was added to $1 \mathrm{~mL}$ of culture suspension and the samples were incubated in the dark at room temperature for $20 \mathrm{~min}$. Finally, $5 \mu \mathrm{L}$ of the stained bacterial suspension was captured between a clean microscope slide and an $18 \mathrm{~mm}$ coverslip. Samples were visualized using a Leica TCS SP5 Confocal Laser Scanning Microscope.

\section{Results and discussion}

Covalent linking of antibacterial agents to surfaces has been considered as a reliable strategy to design antibacterial materials [48] and [49]. We opted here for the covalent linking of a carboxylic acid modified menthol derivative (1) to the hydroxyl groups of ND particles through an esterification reaction with the aim to determine the antibacterial properties of the menthol modified ND particles (Fig. 1). The successful integration of the menthol moiety onto ND was evidenced using Fourier transform infrared spectroscopy (FTIR) (Fig. 2A). ND-OH particles show a broad peak at $3400 \mathrm{~cm}-1$ assigned to the vibration of surface hydroxyl groups or/and adsorbed water molecules, and an additional sharper one at $1633 \mathrm{~cm}-1$ due to the bending mode $\delta(\mathrm{OH})$ of surface hydroxyl groups on the NDs. In addition, the band at $1107 \mathrm{~cm}-1$ is indicative of the presence of C-O-C-functions of cyclic ethers. The FTIR spectrum of the ND-menthol particles displays in addition to bands at 3400 cm- 1 (vibration of surface hydroxyl groups or/and adsorbed water molecules) $1633 \mathrm{~cm}-1$ (bending mode of surface hydroxyl groups) and $1107 \mathrm{~cm}-1$ is (C-O-C- functions of cyclic ethers), a band at 
1717 characteristic of the Cdouble bond; length as m-dashO stretching of the installed ester function. The C-H stretching vibration modes in menthol are seen between 2850 and $2970 \mathrm{~cm}-1$.

(A)

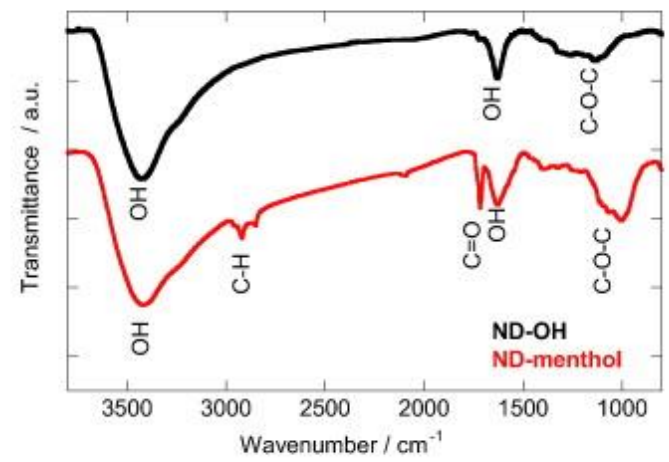

(B)
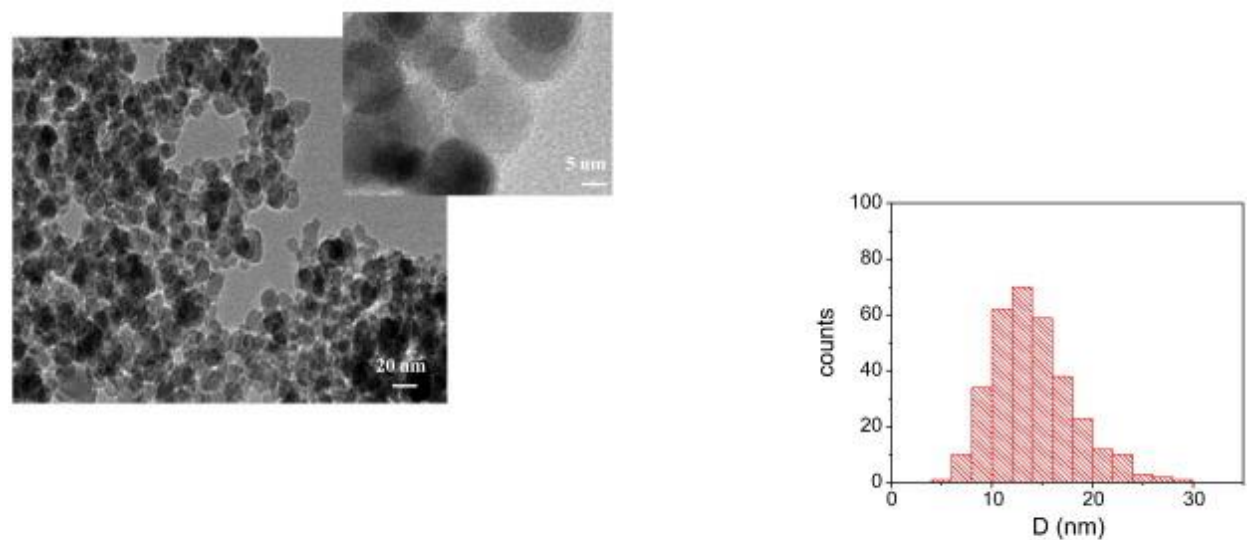

(C)

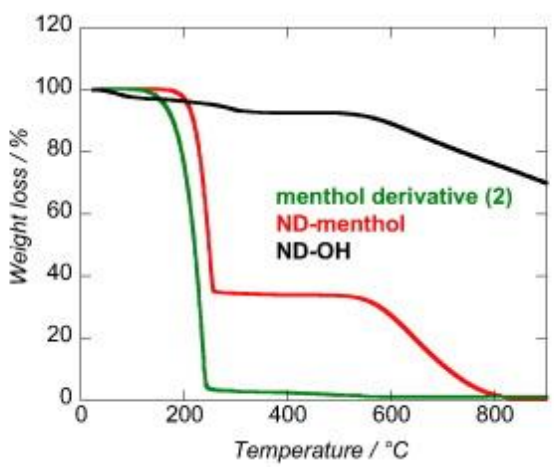

Fig. 2. (A) FTIR spectra of ND-OH (black) and ND-menthol (red); (B) transmission electron microscopy (TEM) images of ND-menthol particles together with histogram of particle size distribution; (C) thermogravimetric analysis of menthol derivative (2) (green), ND-menthol (red) and ND-OH (black).

TEM images of ND-menthol particles (Fig. 2B) reveal the presence of spherical particles with a size distribution in the range of $15 \pm 5 \mathrm{~nm}$ in diameter. The hydrodynamic diameter of ND-menthol particles is $92 \pm 10 \mathrm{~nm}$, comparable to that of ND-OH ( $89 \mathrm{~nm}$ ) with a zeta potential of $29.5 \pm 0.5 \mathrm{mV}$, slightly lower than that of $\mathrm{ND}-\mathrm{OH}(35.3 \pm 1.6 \mathrm{mV})$ (Table 1$)$. 
Table 1. Physico-chemical properties of ND-OH and ND-menthol.

$\begin{array}{llll}\text { ND structure } & \begin{array}{l}\text { Hydrodynamic diameter } \\ (\mathbf{n m})\end{array} & \text { PI } & \begin{array}{l}\text { Zeta potential } \\ (\mathbf{m V})\end{array} \\ \otimes & 89 \pm 13 & 0.246 \pm 0.00235 .3 \pm 1.6 \\ \mathrm{ND}-\mathrm{OH} & 8 \mathrm{OH} & 0.232 \pm 0.01529 .5 \pm 0.5\end{array}$

To gain more insight onto the strength of the bonding between menthol derivative (2) to the surface of ND-OH particles, thermogravimetric analysis of ligand (2), ND-OH and ND-menthol particles was performed (Fig. 2C). The TGA thermogram of the as-received ND-OH particles indicates that they are stable up to $550{ }^{\circ} \mathrm{C}$ without any apparent mass loss [50] and [51]. TGA measurements indicate a weight loss of $\approx 7.45 \%$ above $95^{\circ} \mathrm{C}$ mainly due to absorbed water [50]. Menthol derivative (2) decomposes beyond $\approx 150^{\circ} \mathrm{C}$. The removal of the covalently linked menthol derivative (2) shows its decomposition at temperatures above $\approx 200^{\circ} \mathrm{C}$. The weight loss between 200 and $300{ }^{\circ} \mathrm{C}$ corresponding to the removal of the menthol acid (1) is around $58 \%$ indicating a surface loading of menthol derivative (2) of $2.1 \pm 0.2 \mathrm{mmol} \mathrm{g}-1$.

To test the effect of ND-menthol particles on the growth of pathogens and bacteria induced biofilm formation, Gram-positive S. aureus and Gram-negative E. coli were used as model organisms. S. aureus are extremely versatile pathogenic bacteria that cause a wide range of syndromes, ranging from minor skin and soft tissue infections to life-threatening pneumonia [52] and [53]. Gramnegative E. coli bacteria, widely used as a control organism in microbiological assays, are present within the gastro-intestinal (GI) tract, or as an intra- or extra-intestinal pathogen. A number of antibiotics in use today are delivered orally, and hence the ability of a compound to effect the normal GI flora is of prime importance. Disruption of the Gl flora is not uncommon, but the complete removal of strains or a significant alteration in the relative proportions of the constituent organisms might lead to opportunistic infection by E. coli, or other organisms. Key virulence factors associated with this organism are attachment pili which facilitate adherence to the mucosal lining of the GI or urinary tract [54].

To determine the extent of the antimicrobial activity of ND-menthol particles, they must be assessed against those of known cytotoxic agents to give a positive control. An antibiotic sensitivity test was conducted using a range of 10 common antibiotics at a concentration of $1 \mu \mathrm{g} \mathrm{mL}-1$. The behavior of the different antibiotics towards E. coli growth was determined by the standard microbiological technique of measuring the size of the zone of inhibition around the point where the antibiotic was placed ( Fig. 3). Extremely weak inhibition was observed for penicillin G, while ciprofloxacin and ceftiofur showed relatively strong activity. From the results of the sensitivity test, ampicillin was chosen as a classical antibiotic with an intermediate effect and used for comparison in further experiments. 


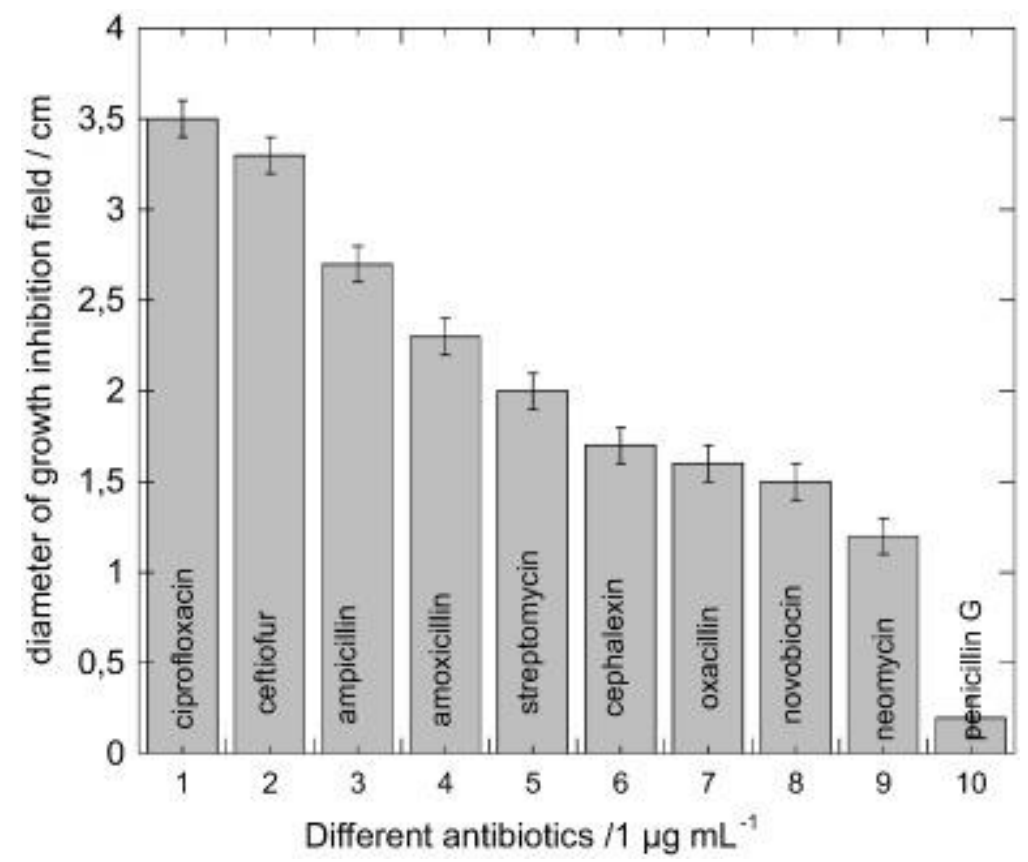

Fig. 3. Antibiotic action on E. coli growth on agar plates.

The planktonic growths of $\mathrm{S}$. aureus and $\mathrm{E}$. coli were thus followed over a 6 hour period in the absence and presence of the ND-menthol particles at different concentrations (Fig. 4). As control the effect of bacterial growth in the presence of unmodified ND-OH $(10 \mu \mathrm{g} \mathrm{mL}-1)$ and ampicillin $(10 \mu \mathrm{g}$ $\mathrm{mL}-1$ ) was investigated. As shown in Fig. 4A, while ND-OH particles do not influence the planktonic growth of $\mathrm{S}$. aureus, ND-menthol particles had a concentration dependent effect on the growth of $\mathrm{S}$. aureus by moderately reducing the growth of the Gram-positive pathogen. However, even at the highest concentration test, the growth of $\mathrm{S}$. aureus was significantly less effective than when an antibiotic was present. In the case of E. coli a similar behavior was observed ( Fig. 4B). While for the first $2 \mathrm{~h}$, E. coli growth was inhibited in the presence of even low concentrations of ND-menthol, their efficiency started to be compromised partially thereafter allowing the planktonic growth of $E$. coli. The effect of ND-menthol on the growth of $\mathrm{S}$. aureus and $\mathrm{E}$. coli was evaluated using a LIVE/DEAD test (Invitrogen) following the manufacturer's instructions. In Fig. 5 show cell growth for S. aureus and $\mathrm{E}$. coli in the absence (control) and presence of ND-menthol $(100 \mu \mathrm{g} / \mathrm{mL})$ and after being stained with the LIVE (green)/DEAD (red) stains. These data suggest that ND-menthol particles do not alter the bacterial viability of S. aureus and E. coli. and are thus non toxic. The toxic effects on membrane structures and function have generally been used to explain the antimicrobial activity of menthol, although the exact mechanism of action is not fully understood [44]. Menthol is believed to alter cell permeability by penetrating between the fatty acid chains making up the membrane lipid bilayers, disrupting lipid packaging and changing membrane fluidity. These phenomena result in major surface alterations and morphological modifications of Gram-positive and Gram-negative bacteria and in leakage of intracellular materials. Linking of menthol to ND particles seems to limit the bactericidal activity of menthol. It is indeed in general believed, that the antibacterial effect of diamond particles is related to its zeta-potential, where particles with positive potential demonstrated no effect, but those with negative zeta potential were shown to disrupt the cell wall and cytoplasmic membrane to release the cytoplasm from the cell [55]. ND-menthol as well as ND$\mathrm{OH}$ particles used as control have both positive zeta-potential (Table 1). More recently, Wehling et al. [37] studied the bactericidal activity of partially oxidized NDs. Their experiments suggested that 
the antibacterial activity of NDs is linked to the presence of partially oxidized and negatively charged surfaces, specifically those containing acid anhydride groups. From the FTIR image of ND-OH and ND-menthol particles (Fig. 2A), the presence of acid anhydride groups with an absorption band at around $1850-1750 \mathrm{~cm}-1$ is not visible explaining in addition to the presence of viable bacteria.

(A)

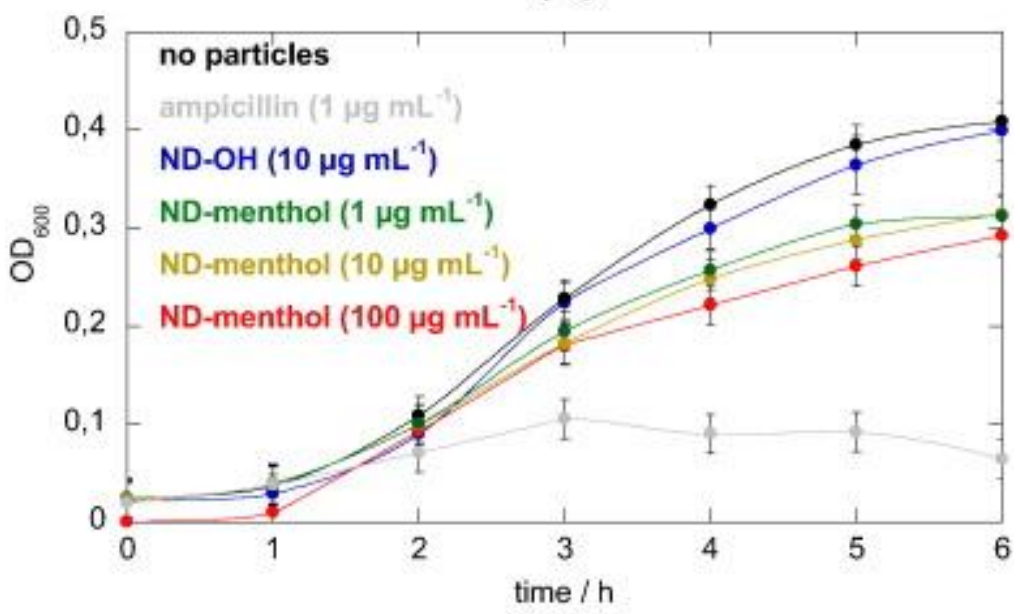

(B)

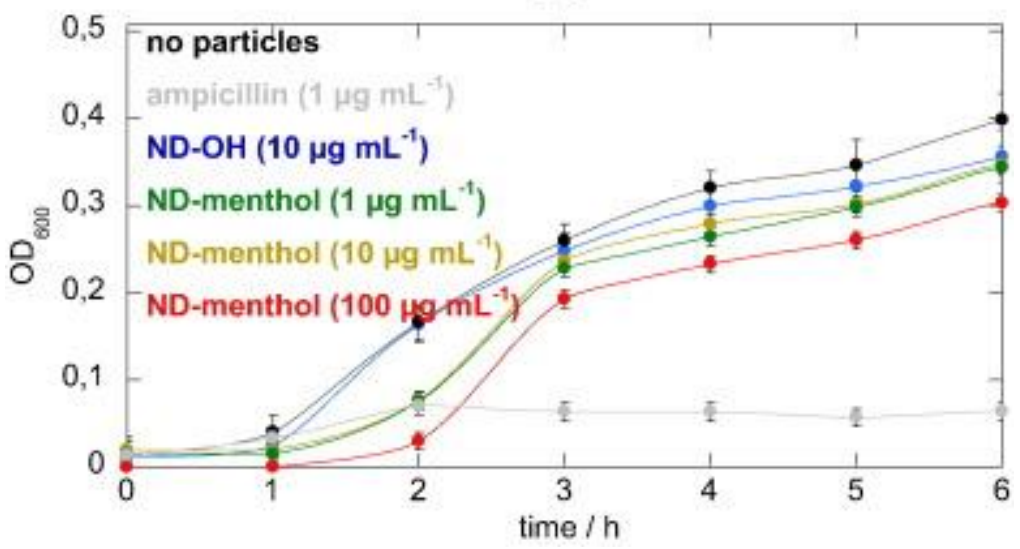

Fig. 4. Growth curves of (A) S. aureus and (B) E. coli in the presence of ND-OH (10 $\mu \mathrm{g} \mathrm{mL}-1)$, ampicillin (1 $\mu \mathrm{g} \mathrm{mL}-1)$ and ND-menthol at 1, 10 and $100 \mu \mathrm{g} \mathrm{m-1}$.

\section{ND-menthol $\left(100 \mu \mathrm{g} \mathrm{mL}^{-1}\right)$}
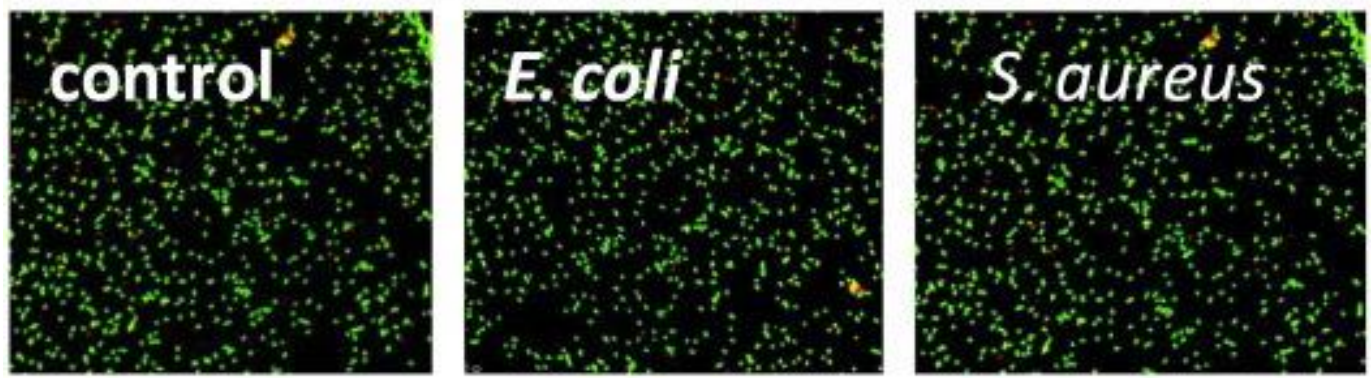

Fig. 5. Viability of S. aureus and E. coli imaged by the LIVE/DEAD ${ }^{\circledR}$ BacLight $^{\mathrm{TM}}$ assay $(\times 40$ magnification) (a) control (no NDs present); (b) ND-menthol. Green fluorescence indicates live cells while red or yellow fluorescence indicates dead or compromised cells. 
While ND-menthol particles were found to not interfer with the development of the planktonic growth of S. aureus and E. coli, we had reasons to believe that ND-menthol particles might affect the adhesion capacitance of the pathogens [47]. The potential of the different nanostructures to inhibit $S$. aureus and $\mathrm{E}$. coli biofilm formation was furthermore investigated. In the case of $\mathrm{S}$. aureus, NDmenthol particles exhibited dose-dependent inhibition of biofilm formation as shown in Fig. 6A. In all cases strong biofilm inhibition is observed with ND-menthol particles. At a particle concentration of $100 \mu \mathrm{g} \mathrm{mL}-1$ the inhibition of biofilm formation was comparable to that of the antibiotic, ampicillin, used. Free menthol ( $100 \mu \mathrm{g} \mathrm{mL}-1)$, on the other hand, did not show any significant biofilm inhibition. ND-OH particles exhibited a moderate biofilm inhibition even at higher concentrations. The reason for the increased biofilm inhibition effect of ND-menthol over free menthol is most likely put down to their more efficient harnessing of a multivalent effect. The difference is probably also driven, in part, by the intrinsic physiochemical properties of the NDs. While more detailed studies are needed to understand fully the antibiofilm activity of menthol modified NDs, the reported change in bacteria membrane fluidity in the presence of menthol [44] seems to reduce the adherence capacity of S. aureus. This is also valid for E. coli biofilms. In the case of E. coli, the NDmenthol particles rival with that of ampicillin even at the lowest concentration level ( Fig. 6B), with high ND-menthol concentrations resulted in strong biofilm inhibition.

\section{Conclusion}

In this work, we have demonstrated that menthol-conjugated nanodiamond particles have marked effects on S. aureus and E. coli-mediated biofilm formation. The conjugation strategy developed based on the formation of an ester bond between the hydroxyl groups of ND and the carboxylic acid group of a synthetic menthol derivative (1) proved to be highly efficient for the coupling of the antibacterial agent. The demonstration that ND-menthol particles are able to effectively impede bacterial adhesion of Gram-positive and Gram-negative bacteria is consistent with our earlier findings that integration of a biofilm inhibitor onto diamond particles has marked E. coli antiadhesive activity. In addition, neither $\mathrm{ND}-\mathrm{OH}$ nor menthol controls are seen to show any antiadhesive activity, underlying that the observed activities are specific to ND-menthol. Moreover, NDmenthol showed better biofilm inhibition than ampicillin even at low particle concentrations. Taken together, the data support that the presence of menthol moiety is the primary ingredient driving the biofilm-inhibitory activity observed for the ND-menthol conjugate. 
(A)

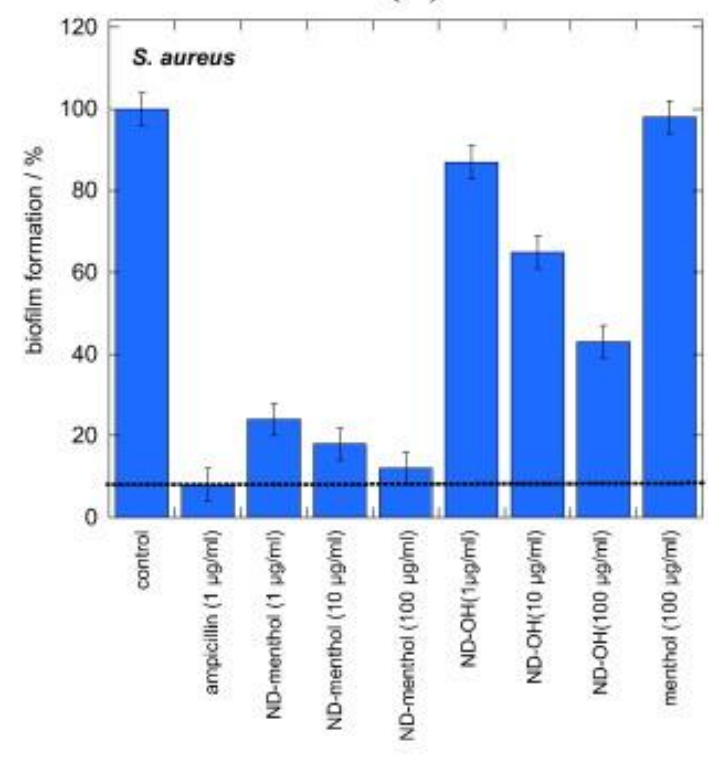

(B)

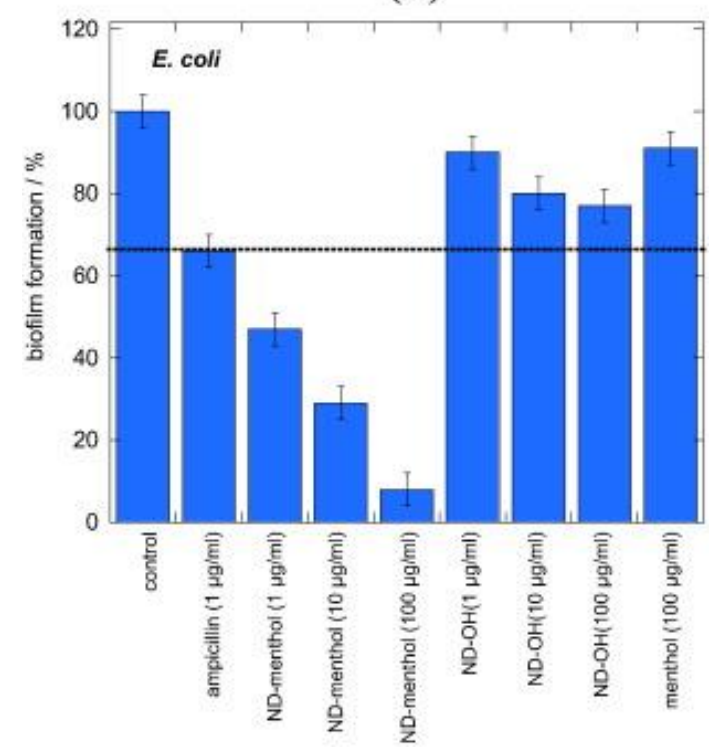

Fig. 6. Biofilm formation of (A) S. aureus and (B) E. coli in the absence and presence of ND-menthol $(1,10,100 \mu \mathrm{g} \mathrm{mL}-1), \mathrm{ND}-\mathrm{OH}(1,10,100 \mu \mathrm{g} \mathrm{mL}-1)$, ampicillin $(1 \mu \mathrm{g} \mathrm{mL}-1)$ and menthol $(100 \mu \mathrm{g} \mathrm{mL}-$ 1).

Prime novelty statement

1. Diamond nanoparticles modified with menthol induce reduction of biofilm formation more efficiently than free menthol.

2. ND-menthol particles also compete with ampicillin, a commonly used antibiotic.

3. Our findings might be thus a step forward towards the development of alternative non-antibiotic based strategies targeting bacterial infections. 


\section{Acknowledgments}

V.T., R.B. and S.S. gratefully acknowledge the financial support from the Centre National de Recherche Scientifique (CNRS), the Université Lille 1, the Nord Pas de Calais region and the Institut Universitaire de France (IUF). R.I. thanks the University of Brighton for a studentship. V.R. and V.C. acknowledge the financial support of the European Commission (IRSES project: 269009). We also acknowledge the support from the European Union through the FP7-PEOPLE-2010-IRSES action "Photorelease" (grant number 269099). R. J. thanks Institut Français in Romania for a PhD scholarship. We thank Profs P. Woisel, J. Lyskawa and A. Malfait (UMET UMR CNRS 8207, Université de Lille Nord de France, ENSCL) for the technical support with TGA measurements.

\section{References}

[1] A. Barras, F.A. Martin, O. Bande, J.S. Baumann, J.-M. Ghigo, R. Boukherroub, C. Beloin, A. Siriwardena, S. Szunerits, Glycan-functionalized diamond nanoparticles as potent E. coli antiadhesives, Nanoscale, 5 (2013), p. 2307

[2] M. Durka, K. Buffet, J. lehl, M. Holler, J.F. Nierengarten, J. Taganna, J. Bouckaert, S.P. Vincent, The functional valency of dodecamannosylated fullerenes with Escherichia coli FimH-towards novel bacterial antiadhesives, Chem. Commun., 47 (2011), p. 1321

[3] P. Laurino, R. Kikkeri, N. Azzouz, P.H. Seeberger, Detection of bacteria using glyco-dendronized polylysine prepared by continuous flow photofunctionalization, Nano Lett., 11 (2011), p. 73

[4] P.C. Ray, S.A. Khan, A.K. Singh, D. Senapati, Z. Fan, Nanomaterials for targeted detection and photothermal killing of bacteria, Chem. Soc. Rev., 41 (2012), p. 3193

[5] A. Herman, A.P. Herman, Nanoparticles as antimicrobial agents: their toxicity and mechanisms of action, J. Nanosci. Nanotechnol., 14 (2014), pp. 946-957

[6] R.P. Allaker, K. Memarzadeh, Nanoparticles and the control of oral infections, Int. J. Antimicrob. Agents, 43 (2014), pp. 95-104

[7] S. Chernousova, M. Epple, Silver as antibacterial agent: ion, nanoparticle, and metal, Angew. Chem. Int. Ed., 52 (2012), pp. 1636-1653

[8] M.R. Das, R.K. Sarma, R. Saikia, V.S. Kale, M.V. Shelke, P. Sengupta, Synthesis of silver nanoparticles in an aqueous suspension of graphene oxide sheets and its antimicrobial activity, Colloids Surf. B Biointerfaces, 83 (2011), p. 16

[9] Y. Zhao, Y. Tian, Y. Cui, L. Wenwen, W. Ma, X. Jiang, Small molecule-capped gold nanoparticles as potent antibacterial agents that target gram-negative bacteria, J. Am. Chem. Soc., 132 (2010), p. 12349

[10] O. Akhavan, R. Azimirad, S. Safed, E. Hassani, $\mathrm{CuO} / \mathrm{Cu}(\mathrm{OH}) 2$ hierarchical nanostructures as bactericidal photocatalysts, J. Mater. Chem., 21 (2011), p. 9634 
[11] G. Qi, L. Li, F. Yu, H. Wang, Vancomycin-modified mesoporous silica nanoparticles for selective recognition and killing of pathogenic gram-positive bacteria over macrophage-like cells,ACS Appl. Mater. Interfaces, 5 (2013), p. 10874

[12] R.Y. Pelgrift, A.J. Friedman, Nanotechnology as a therapeutic tool to combat microbial resistance, Adv. Drug Deliv. Rev., 65 (2013), pp. 1803-1815

[13] K. Forier, K. Raemdonck, S.D. De Smedt, J. Demeester, T. Coenye, K. Braeckmans, Lipid and polymer nanoparticles for drug delivery to bacterial biofilms, J. Control. Release, 190 (2014), pp. 607-623

[14] X. Zhu, A.F. Radovic-Moreno, J. Wu, R. Langer, J. Shi, Nanomedicine in the management of microbial infection - overview and perspectives, Nano Today, 9 (2014), pp. 478-498

[15] P. Ma, R.J. Mumper, Anthracycline nano-delivery systems to overcome multiple drug resistance: a comprehensive review, Nano Today, 8 (2013), p. 313

[16] M.R. Das, R.K. Sarma, S.C. Borah, R. Kumri, R. Saikia, A.B. Deshmukh, M.V. Shelke, P. Sengupta, $S$. Szunerits, R. Boukherroub, Synthesis of silver nanoparticles in an aqueous suspension of graphene oxide sheets and its antimicrobial activity, Colloids Surf. B, 105 (2013), pp. 128-136

[17] J.S. Kim, E. Kuk, K.N. Yu, J.H. Kim, S.J. Park, H.J. Lee, S.H. kim, Y.K. Park, Y.H. Park, C.Y. Hwang, Y.K. Kim, Y.S. Lee, D.H. Jeong, M.H. Cho, Antimicrobial effects of silver nanoparticles, Nanomedicine, 3 (2007), pp. 95-101

[18] S. Mohanty, S. Mishra, P. Jena, B. Jacob, B. Sarkar, A. Sonawane, An investigation on the antibacterial, cytotoxic, and antibiofilm efficacy of starch-stabilized silver nanoparticles, Nanomedicine, 8 (2012), pp. 916-924

[19] J.-H. Liu, D.A. Sonshine, S. Shervani, R.H. Hurt, Controlled release of biologically active silver from nanosilver surfaces, ACS Nano, 4 (2010), p. 6903

[20] A. Panacek, L. Kvitek, R. Prucek, M. Kolar, R. Vecerova, N. Pizurova, V.K. Sharma, T. Nevecna, R. Zboril, Silver colloid nanoparticles: synthesis, characterization, and their antibacterial activity, J. Phys. Chem. B, 110 (2006), p. 16248

[21] S. Pal, Y.K. Tak, J.M. Song, Does the antibacterial activity of silver nanoparticles depend on the shape of the nanoparticle? A study of the gram-negative bacterium Escherichia coli, Appl. Environ. Microbiol., 73 (2007), pp. 1712-1720

[22] K.K.Y. Wong, X. Liu, Silver nanoparticles-the real "silver bullet" in clinical medicine, Med. Chem. Commun., 1 (2010), pp. 125-131

[23] H. Kong, J. Jang, Antibacterial properties of novel poly(methyl methacrylate) nanofiber containing silver nanoparticles, Langmuir, 24 (2008), pp. 2051-2056

[24] A. Barras, J. Lyskawa, S. Szunerits, P. Woisel, R. Boukherroub, Direct functionalization of nanodiamond particles using dopamine derivatives, Langmuir, 27 (2011), p. 12451 
[25] A. Barras, S. Szunerits, L. Marcon, N. Monfilliette-Dupont, R. Boukherroub, Functionalization of diamond nanoparticles using "click" chemistry, Langmuir, 26 (2010), pp. 13168-13172

[26] Y.-R. Chang, H.-Y. Lee, K. Chen, C.-C. Chang, D.-S. Tsai, C.-C. Fu, T.-S. Lim, Y.-K. Tzeng, C.-Y. Fang, C.-C. Han, H.-C. Chang, W. Fann, Mass production and dynamic imaging of fluorescent nanodiamonds, Nat. Nanotechnol., 3 (2008), pp. 284-288

[27] S.A. Dahoumane, M.N. Nguyen, A. Thorel, J.P. Boudou, M.M. Chehimi, C. Mangeney, Proteinfunctionalized hairy diamond nanoparticles, Langmuir, 25 (2009), pp. 9633-9638

[28] A. Krüger, Hard and soft: biofunctionalized diamond, Angew. Chem. Int. Ed., 45 (2006), pp. 6426-6427

[29] A. Krüger, New carbon materials: biological applications of functionalized nanodiamond materials, Chem. Eur. J., 14 (2008), pp. 1382-1390

[30] Y. Liang, M. Ozawa, A. Krueger, A general procedure to functionalize agglomerating nanoparticles demonstrated on nanodiamond, ACS Nano, 3 (2009), pp. 2288-2296

[31] V.N. Mochalin, O.A. Shenderova, D. Ho, Y. Gogotsi, The properties and applications of nanodiamonds, Nat. Nanotechnol., 7 (2012), p. 11

[32] L. Marcon, F. Riquet, D. Vicogne, S. Szunerits, J.-F. Bodart, R. Boukherroub, Cellular and in vivo toxicity of functionalized nanodiamond in Xenopus embryos, J. Mater. Chem., 20 (2010), pp. 8064 8069

[33] K.K. Liu, C.L. Cheng, C.C. Chang, J.I. Chao, Biocompatible and detectable carboxylated nanodiamond on human cell, Nanotechnology, 18 (2007), p. 325102

[34] A.M. Schrand, H. Huang, C. Carlson, J.J. Schlager, E. Omacr Sawa, S.M. Hussain, L. Dai, Are diamond nanoparticles cytotoxic?, J. Phys. Chem. B, 111 (2007), pp. 2-7

[35] S.J. Yu, M.W. Kang, H.C. Chang, K.M. Chen, Y.C. Yu, Bright fluorescent nanodiamonds: no photobleaching and low cytotoxicity, J. Am. Chem. Soc., 127 (2005), pp. 17604-17605

[36] E. Perevedentseva, C.-Y. Cheng, P.-H. Chung, J.-S. Tu, T.-H. Hsieh, C.-L. Cheng, The interaction of the protein lysozyme with bacteria E. coli observed using nanodiamond labelling, Nanotechnology, 18 (2007), p. 315107

[37] J. Wehling, R. Dringer, R.N. Zare, M. Maas, K. Rezwan, Bactericidal activity of partially oxidized nanodiamonds, ACS Nano, 8 (2014), p. 6475

[38] M. Hartmann, P. Betz, Y. Sun, S.N. Gorb, T.K. Lindhorst, A. Krueger, Saccharide-modified nanodiamond conjugates for the efficient detection and removal of pathogenic bacteria, Chem. Eur. J., 18 (2012), p. 6485

[39] X.R. Wu, T.T. Sun, J.J. Medina, In vitro binding of type 1-fimbriated Escherichia coli to uroplakins la and Ib: relation to urinary tract infections, Proc. Natl. Acad. Sci. U. S. A., 93 (1996), pp. 9630-9635 
[40] C.-C. Lin, Y.-C. Yeh, C.-Y. Yang, C.-L. Chen, G.-F. Chen, C.-C. Chen, Y.-C. Wu, Selective binding of mannose-encapsulated gold nanoparticles to type 1 pili in Escherichia coli, J. Am. Chem. Soc., 124 (2002), p. 3508

[41] T. Patel, Y. Ishiuji, G. Yosipovitch, Menthol: a refreshing look at this ancient compound, J. Am. Acad. Dermatol., 57 (2007), pp. 873-878

[42] J.A. Farco, O. Grundmann, Menthol - pharmacology of an important naturally medicinal "cool", Mini-Rev. Med. Chem., 13 (2013), pp. 124-131

[43] G.P.P. Kamatou, I. Vermaak, A.M. Viljoen, B.M. Lawrence, Menthol: a simple monoterpene with remarkable biological properties, Phytochemistry, 96 (2013), pp. 15-25

[44] D. Trombetta, F. Castelli, M.G. Sarpietro, V. Venuti, M. Christiani, C. Daniele, A. Saija, G. Mazzanti, G. Bisignani, Mechanism of antibacterial action of three monoterpenes, Antimicrob. Agents Chemother., 49 (2005), pp. 2474-2478

[45] G. Iscan, N. Kirimer, M. Kurkcuoglu, K.H. Baser, F. Demirci, Antimicrobial screening of Mentha piperita essential oils, J. Agric. Food Chem., 50 (2002), pp. 3943-3946

[46] R. Kotan, S. Kordali, A. Cakir, Screening of antibacterial activities of twenty-one oxygenated monoterpenes, Z. Naturforsch. C, 62 (2007), pp. 507-513

[47] S. Dalleau, E. Cateau, T. Bergès, J.-M. Berheaud, C. Imbert, In vitro activity of terpenes against Candida biofilms, Int. J. Antimicrob. Agents, 31 (2008), pp. 572-576

[48] A. Tiraferri, C.D. Vecitis, M. Elimelech, Covalent binding of single-walled carbon nanotubes to polyamide membranes for antimicrobial surface properties, ACS Appl. Mater. Interfaces, 3 (2011), pp. 2869-2877

[49] S.A. Al-Bataineh, R. Luginbuehl, M. Textor, M. Yan, Covalent immobilization of antibacterial furanones via photochemical activation of perfluorophenylazide, Langmuir, 25 (2009), pp. 74327437

[50] A. Krüger, Y. Liang, G. Jarre, J. Stegk, Surface functionalisation of detonation diamond suitable for biological applications, J. Mater. Chem., 16 (2006), p. 2322

[51] V. Pichot, M. Comet, E. Fousson, C. Baras, A. Senger, F. Le Normand, D. Spitzer, An efficient purification method for detonation nanodiamonds, Diamond Relat. Mater., 17 (2008), p. 13

[52] D.J. Diekema, M.A. Pfaller, F.J. Schmitz, J. Smayevsky, J. Bell, R.N. Jones, M. Beach, Survey of infections due to Staphylococcus species: frequency of occurrence and antimicrobial susceptibility of isolates collected in the United States, Canada, Latin America, Europe, and the Western Pacific Region for the SENTRY Antimicrobial Surveillance Program, 1997-1999, Clin. Infect. Dis., 32 (2001), pp. S114-S132

[53] E. Boakes, A.M. Kearns, M. Ganner, C. Perry, R.L. Hill, M.J. Ellington, Distinct bacteriophages encoding panton-valentine leukocidin (PVL) among international methicillin-resistant Staphylococcus aureus clones harboring PVL, J. Clin. Microbiol., 49 (2010), pp. 684-692 
[54] J.P. Zhang, S. Normark, Induction of gene expression in Escherichia coli after pilus-mediated adherence, Science, 273 (1996), pp. 1234-1236

[55] A. Chwalibog, E. Sawosz, A. Hotowy, J. Szeliga, S. Mitura, K. Mitura, M. Grodzik, P. Orlowski, A. Sokolowska, Visualization of interaction between inorganic nanoparticles and bacteria or fungi, Int. J. Nanomedicine, 5 (2010), pp. 1085-1094 